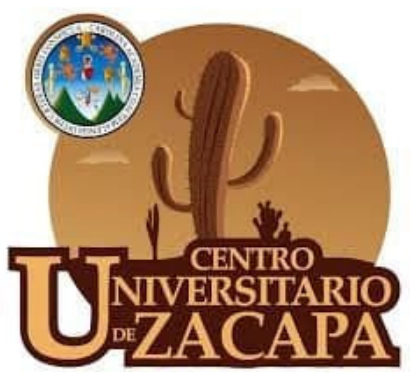

Recibido: $16 / 11 / 2020$

Ensayo científico

Publicado: 15/01/2021

\title{
Abordaje multidisciplinario del síndrome de sun- downing en deterioro neurocognitivo
}

\section{Multidisciplinary approach to sundowning syndrome in neuro- cognitive impairment}

\author{
Héctor Alfredo René Bran Girón \\ Mario Josué Lopéz Rojas \\ Josué Elizandro Pinto Cabrera \\ Nataly Mercedes Chó Paiz \\ Ingrid Estefanía Linares Pinto \\ Edwin Orlando Lucas Vanegas
}

Filiación institucional de los autores

Universidad de San Carlos de Guatemala

hector.bran198720141@gmail.com

https://orcid.org/0000-0002-4487-2252

\section{Referencia del ensayo}

Bran Girón, H. A., López Rojas, M. J., Pinto Cabrera, J. E., Chó Paiz, N. M., Linares Pinto, I. E. y Lucas Vanegas, E. O. (2021). Abordaje multidisciplinario del síndrome de sundowning en deterioro neurocognitivo. Revista Académica CUNZAC, 4(1). 17-24. DOI: https://doi.org/10.46780/cunzac.v4i1.27

\section{Resumen}

Las neurociencias son vitales para la atención de las personas mayores a partir de los sesenta años y más, debe ser orientada en la prevención, terapéutica, neurorehabilitación y abordaje de cuidados paliativos encaminados para brindar un soporte en el cuidado de la persona mayor enferma en etapas agudas cuando surge la aparición del síndrome de sundowning que significa el deterioro de funciones cognitivas relacionadas con el trastornos del sueño y el aparecimiento de síntomas dependiendo la jornada nocturna y diurna. Por consiguiente, en la población geriátrica de manera fisiológica se activa el ciclo circadiano del sueño durante el atardecer para la conciliación normal del mismo; es aquí cuando aparece el síndrome de sundowning de manera patológica caracterizándose por un conjunto de signos y síntomas que se presentan en pacientes con deterioro neurocognitivo. Este síndrome debe abordarse de manera multidisciplinaria debido a que esta agrupación de perturbaciones del comportamiento tanto emocionales, físicas y cognitivas son derivadas de las diferentes demencias aunadas a los grandes síndromes geriátricos que agudizan el cuadro clínico tornando a los pacientes con aumento de los grados de dependencia y fragilidad resultado del deterioro de la calidad de vida, bienestar físico y mental del adulto mayor.

Palabras clave: sundowning, neurocognición, multidisciplinariedad, abordaje, demencia

\begin{abstract}
Neurosciences are vitals for the care of the elderly from the age of sixty and over, it must be oriented in the prevention, therapy, neurorehabilitation and approach of palliative care aimed at providing support in the care of the sick elderly person in stages acute when the appearance of sundowning syndrome arises, which means the deterioration of cognitive functions related to sleep disorders and the appearance of symptoms depending on the night and day shift. Consequently, in the geriatric population the circadian cycle of sleep is physiologically activated during the evening for normal conciliation of the same; This is when the sundowning syndrome appears pathologically, characterized by a set of signs and symptoms that occur in patients with neurocognitive impairment. This syndrome must be approached in a multidisciplinary way because this grouping of emotional, physical and cognitive behavioral disturbances are derived from the different dementias coupled with the large geriatric syndromes that exacerbate the clinical picture, making patients with increased degrees of dependence and frailty as a result of the deterioration of the quality of life, physical and mental well-being of the elderly.
\end{abstract}

Keywords: sundowning, neurocognition, multidisciplinarity, approach, dementia

\begin{tabular}{l|l|l|l|l|l|l|l|l}
\hline CUNZAC & Revista del Centro Universitario de Zacapa & ISSN: 2708-7158 & Volumen 4 & Junio 2021 \\
\hline
\end{tabular}




\section{Introducción}

El término demencia se refiere al grupo de patologías de neurodegeneración de índole progresiva que afecta a la neurocognición, según la Organización Mundial de la Salud la demencia se integra en este grupo caracterizándose por el inicio temprano que se manifiesta antes de los 60 años; evidenciando la disminución o pérdida de habilidades neurocognitivas.

Un reporte de World Alzheimer Report en el 2018 indicó que a nivel mundial 50 millones de personas fueron diagnosticadas con demencia y se pronostica que para el año 2030 los diagnósticos se tripliquen. Es por ello que las personas con demencia manifiestan un comportamiento alterado en horas específicas del atardecer y anochecer; entre las manifestaciones surgen desorientación, agitación, confusión, inquietud y comportamientos agresivos que preocupan a los cuidadores denominado síndrome del ocaso (Assad, Ghani, Sulehria, Mansoor, \& Ameer, 2017).

A nivel mundial el crecimiento poblacional y la frecuencia del envejecimiento patológico es evidente siendo el aparecimiento del deterioro cognitivo una entidad sumamente importante en el estudio de la salud y el abordaje multidisciplinario.

El presente estudio permite describir de manera científica e informativa el abordaje multidisciplinario del síndrome de sundowning en pacientes con demencia siendo capaces de identificar la severidad de los síntomas que conforman la definición del mismo y reconocer el abordaje individual y a la vez integral que promueve un enfoque holístico y multidisciplinario mediante el estudio de las neurociencias que brindan inclusión, calidad de vida y efectividad de atención a personas mayores con deterioro de la cognición.

El objetivo es establecer el enfoque de las neurociencias en el abordaje integral del síndrome de sundowning en pacientes con deterioro neurocognitivo con el fin de conocer la enfermedad asociada y su relación con los grandes síndromes geriátricos mediante el fundamento de neurociencias.

\section{Contenido}

En neurofisiología se conoce el término zeitgeber y el ciclo circadiano del sueño que está en la normalidad estos se vinculan con los dos núcleos identificados en el SNC, como los marcapasos del periodo circadiano se encuentran, el núcleo supraquiasmático (NSC) se encuentra en el hipotálamo ventral y una región en el núcleo ventromedial del hipotálamo (HVM). Estos son los encargados de la regulación del ciclo vigilia y sueño entre otras funciones fisiológicas (Ravi \& Bass, 2021).

Los pacientes con síndrome de Sundowning pueden llegar a presentar alteraciones del ciclo circadiano o reloj biológico del estado de vigilia-sueño, consecuencia de la inhibición o baja producción de hormonas esenciales para llevar a cabo dichos procesos.

En pacientes con alteraciones hormonales se pueden identificar un claro incremento de los despertares tanto en duración del estado de vigilia y la frecuencia de dicho estado, lo que a su vez conlleva a un incremento de la cantidad de siestas en horario matutino y vespertino. La melatonina es una neurohormona fundamental en la fisiología circadiana ya que es una reguladora hormonal en el ritmo circadiano (lbid). 
A nivel de estudios electroencefalográficos durante el sueño se detecta una disminución del sueño lento profundo en la fase 3-4 del sueño no-REM y REM, lo que nos lleva a la principal manifestación clínica de dichas alteraciones siendo el insomnio.

Figura No. 1 Zeitgeber y reloj biológico

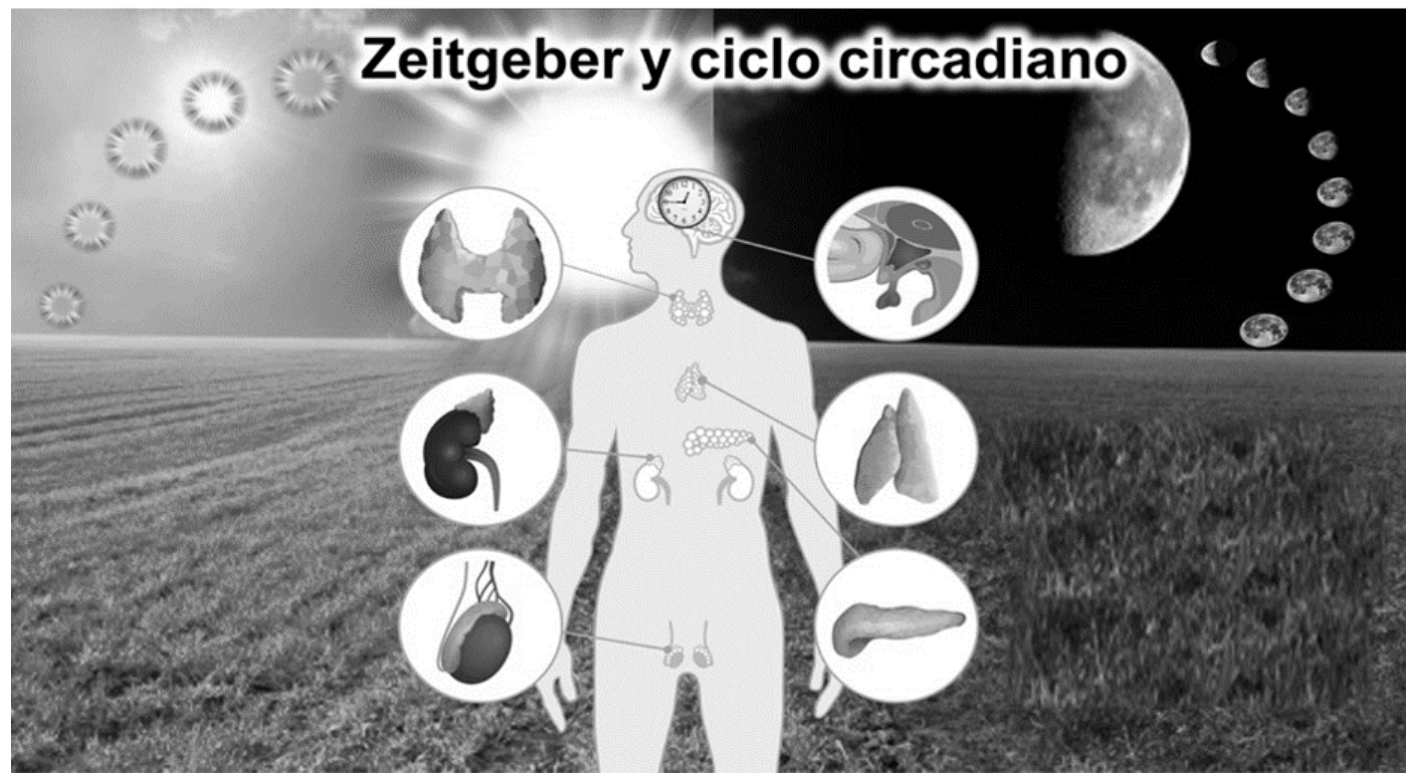

Fuente: propia. Con información con base del artículo Potential Pathways for Circadian Dysfunction and Sundowning-Related Behavioral Aggression in Alzheimer's Disease and Related Dementias.

El síndrome de sundowning como se mencionó con anterioridad afecta el ciclo circadiano del sueño que se manifiesta en la dependencia de horario tanto en jornadas nocturnas y diurnas; este proceso se tipifica en pacientes con deterioro neurocognitivo en fases de demencias correlacionadas con score GDS, identifica la demencia de manera global.

La demencia representa actualmente un problema latente en la salud pública. Involucra el cese de funciones cognitivas, generando discapacidades cognitivas y anatómicas funcionales, y con ello mayor dependencia de las acciones cotidianas. Se dispone de información epidemiológica escasa que revele un valor de su prevalencia en el grupo de adultos mayores.

Los trastornos del sueño son muy frecuentes en los adultos mayores con demencia, tiene gran importancia el conocimiento de las enfermedades del sueño que les afectan y cuál debe ser su tratamiento. El síndrome de sundowning tiene una importante repercusión social por las alteraciones del estado de conciencia caracterizada por delirio, confusión temporoespacial, pensamiento desorga- 
nizado, alucinaciones, inatención, inquietud, hiperactividad manifestada por deambulación constante en los espacios habitacionales, agitación o irritabilidad, insomnio y ansiedad que se desarrollan en las últimas horas de luz solar.

El síndrome de sundowning afecta los procesos mentales encargados de interpretar, crear y transmitir información; aumentando la gravedad de su disfunción con mayor dificultad en horas específicas del atardecer debido a las alteraciones que se vinculan entre el núcleo supraquiasmático asociado al sistema circadiano (Llanes et. al., 2019).

Figura No. 2 Demencia y Disfunción neurocognitiva global

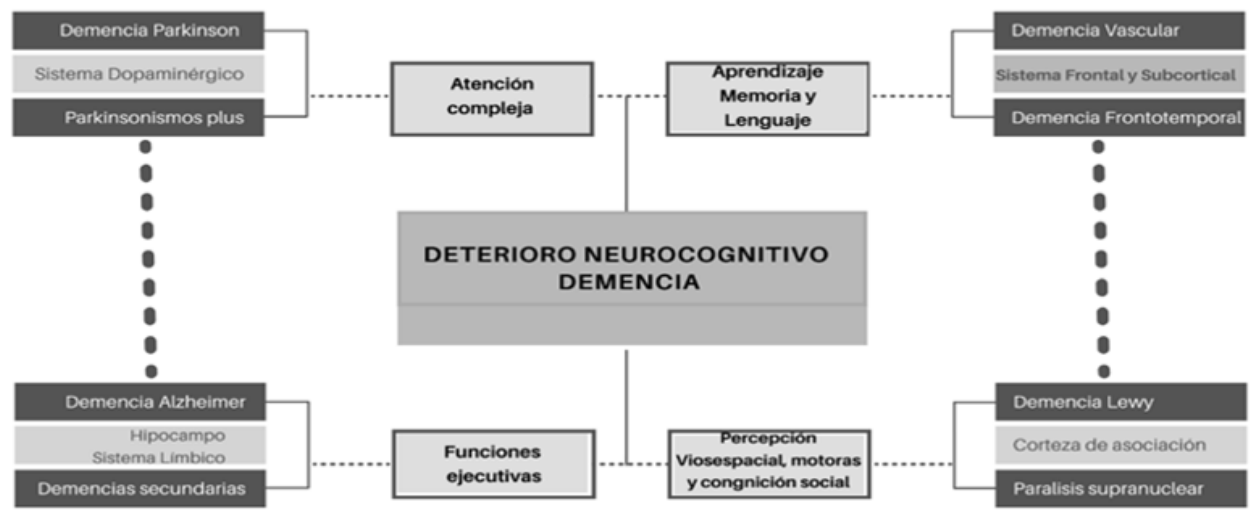

Fuente: elaboración propia. Con base al Manual de geriatría para médicos.

La dependencia se presenta como una circunstancia de asistencia y apoyo de otras personas, debido a problemas físicos, psíquicos o intelectuales. Por lo que se pierde la autonomía de actividades personales esenciales. Las cuales presentan las siguientes características como la disminución de capacidades personales por las limitaciones presentadas, no poder realizar actividades cotidianas y el apoyo de cuidados especiales por parte de cuidadores.

El índice de Barthel (IB) es una valoración geriátrica que evalúa las actividades de la vida diaria determinando el grado de dependencia que refleja el abordaje de los cuidadores pri- marios y secundarios asimismo como el enfoque multidisciplinario en el plan ambulatorio o en residencias geriátricas, este índice sigue siendo una herramienta factible y adecuada para trazar las metas claves de dar calidad de vida y reducir el avance de la dependencia como tal.

La fragilidad tiene las características de presentar un decrecimiento en la resistencia y un desgaste fisiológico. Teniendo como consecuencias caídas, las cuales pueden requerir de una hospitalización o internar en un asilo por no tener la capacidad de cuidados en casa. Se puede presentar de discapacidades o en el peor de los casos hasta la muerte. La Enero - Junio 2021 
prevalencia reportada oscila entre un 7 y un $12 \%$ en la población mayor de 65 años y se asocia en pacientes con trastornos psicogeriátricos debido a la afección cognitiva y se corrobora con la escala de Rockwood modificada. La neurociencia tiene como finalidad el estudio del sistema nervioso enfocados al comportamiento y la conducta humana, es por ello la necesidad de conocer el abordaje médico, psicológico y fisioterapéutico para abordar de manera efectiva el síndrome de sundowning en los pacientes con deterioro neurocognitivo. Este abordaje debe ser completamente integral porque cada disciplina forma parte de una valoración geriátrica ética en los adultos mayores con deterioro neurocognitivo.

El abordaje médico desde la perspectiva de neurociencias médicas es totalmente interesante siendo el eje para el diagnóstico oportuno es necesaria la formación de geriatras, neurólogos y médicos en neurociencias, debido a la atención en el nivel preventivo, diagnóstico y tratamiento y el proceso de neurorrehabilitación. Los esfuerzos del manejo médico son evidentes mediante la valoración geriátrica en el que se desarrolla el análisis del cuadro clínico, parámetros de laboratorio para descartar deterioro neurocognitivo desde la neurodegeneración primaria versus secundaria dotando de parámetros bioquímicos como los niveles de vitamina $D$ y melatonina que son predictores de fragilidad, dependencia y deterioro neurocognitivo.

El tamizaje bioquímico y genético entre ellos coenzima Q10, alfasinucleína, ubiquitina, B42 amiloide, proteína Tau y fosforilasa Tau, asimismo el diagnóstico fino mediante la medición volumétrica de estructuras específicas en estudios de neuroimagen como la resonancia magnética cerebral de cortes coronales y transversales con énfasis hipocampal y estudios de medicina nuclear como lo son el SPECT cerebral. El abordaje de neurociencias médicas va dirigido a un diagnóstico acertado con el objetivo de dirigir el tratamiento oportuno específico para cada tipo de demencia asociada al síndrome de sundowning.

Tabla No.1 Neurociencias médicas en Sundowning y Demencia

\begin{tabular}{|c|c|c|c|}
\hline \multirow[b]{2}{*}{ Tipo de Demencia } & \multicolumn{3}{|c|}{ Parkinson } \\
\hline & Frontotemporal & Cuerpos de Lewy & Alzheimer \\
\hline $\begin{array}{l}\text { Ubicación } \\
\text { neuroanatómica }\end{array}$ & Sistema fronto-subcortical & $\begin{array}{l}\text { Sistema dopaminérgico y } \\
\text { límbico }\end{array}$ & $\begin{array}{l}\text { Hipocampo } \\
\text { Corteza de asociación }\end{array}$ \\
\hline Cuadro Clínico & $\begin{array}{l}\text { Disfunción ejecutiva y cambios de la } \\
\text { personalidad, Desinhibición y apatía }\end{array}$ & $\begin{array}{l}\text { Trastornos en atención } \\
\text { Habilidad visoespacial, } \\
\text { depresión, trastornos del } \\
\text { sueño y alucinaciones }\end{array}$ & $\begin{array}{l}\text { Amnesia, afasia, psicosis y } \\
\text { depresión }\end{array}$ \\
\hline Proteinopatías & Tau / Ubiquitina & Sinucleína & $\begin{array}{l}\text { Tau / Fosforilasa Tau / B } \\
\text { amiloide }\end{array}$ \\
\hline $\begin{array}{l}\text { Estudios de } \\
\text { neuroimagen }\end{array}$ & $\begin{array}{l}\text { Resonancia magnética cerebral y } \\
\text { SPECT cerebral } \\
\text { Atrofia cortical frontotemporal }\end{array}$ & $\begin{array}{l}\text { Resonancia magnética } \\
\text { cerebral y SPECT cerebral } \\
\text { Ganglios basales }\end{array}$ & $\begin{array}{l}\text { Resonancia magnética } \\
\text { cerebral y SPECT cerebral } \\
\text { Ovillos neurofibrilares y } \\
\text { destrucción hipocampal }\end{array}$ \\
\hline
\end{tabular}

Fuente: elaboración propia. con base al Manual de geriatría para médicos.

Volumen 4 | Número 1 |Enero - Junio 2021 
Desde la perspectiva neuropsicológica las enfermedades neurodegenerativas los síntomas conductuales representan una mayor cantidad de atención por parte de la familia, como lo expresa (García-Díaz, 2020); provocando que la dinámica familiar se vea afectada, conllevando a tener que utilizar el apoyo de terceras personas (cuidadores, instituciones hospitalarias o centros de atención para adultos mayores).

Es por ello de la importancia de la intervención del profesional de la salud mental, así como también significa un reto para los psicólogos, dado que dichas enfermedades pueden empeorar notablemente en poco tiempo, llevando a los pacientes a un deterioro mayor, provocando que disminuya su independencia y su calidad de vida.

La valoración psicogerontológica es fundamental en la evaluación del estado mental del adulto mayor mediante el Mini Mental State Examination (MMSE) modificado para personas de edad que tiene como objetivo identificar el grado de deterioro neurocognitivo en leve, moderado y severo teniendo una sensibilidad de $92.3 \%$ y especificidad de $53.7 \%$ (Alvarado, et. al., 2019).

Una segunda herramienta posterior a la evaluación del estado mental del adulto mayor es identificar la Escala de Deterioro Global (GDS) de Reisberg siendo el objetivo clave la relación del deterioro neurocognitivo con las manifestaciones del sundowning teniendo una sensibilidad de $95.7 \%$ y especificidad de 92.4\%; la valoración geriátrica integral desde la neurociencia y psicología no solo se encarga de identificar el deterioro neurocognitivo es así como se suman el análisis de las actividades básicas, instrumentales y avanzadas que son necesarias manejarlas en consenso secundario al aparecimiento de fragilidad, de- pendencia y otros síndromes geriátricos asociados. (Ibid.)

El objetivo de esta valoración es trazar metas claves para el psicólogo en la realización de estrategias neurocognitivas integradas utilizadas en clínicas de memoria, neurorrehabilitación sensorial que abarcan y el plan educacional para el cuidador primario y secundario desde la neurocognición social y clínica.

En conjunto el enfoque de la fisiogeriatria es un campo multidisciplinario vital en el síndrome de sundowning derivado de la aparición del cuadro clínico con afección neurocognitiva son los factores desencadenantes del incremento del estado de fragilidad y dependencia.

Los pacientes que presentan esta etiología necesitan un abordaje neurocientífico dirigido a la neurorehabilitación siendo de gran valor la identificación de síndromes vinculados mediante una valoración fisioterapéutica integral siendo los factores determinantes las actividades de la vida diaria siendo medidas por el índice Barthel referente a las básicas que tienen una sensibilidad de $88 \%$ y una especificidad del $98 \%$ y las clasificadas como instrumentales mediante la escala de Lawton Brody con un valor de sensibilidad de la $94 \%$ y específica de $82 \%$.

Para el fisioterapeuta la dependencia se relaciona con la fragilidad estas dos grandes entidades se vínculan con el deterioro neurocognitivo y el aparecimiento de los síntomas del sundowning es por ello la necesidad de evaluar la escala de Rockwood modificada que es una prueba sensible de $78 \%$ y especifica del $85 \%$ permitiendo valorar tres posibles causas de morbimortalidad en los adultos mayores con sundowning. 
En la actualidad se presenta un incremento en la cantidad de adultos mayores, lo cual es de importancia para poder brindar una atención de calidad, ya que muchos de ellos no cuentan con el apoyo de sus familias. Las técnicas fisioterapueticas deben ir encaminadas en mejorar el estado de dependencia y fragilidad asimismo contribuir a controlar el síndrome de sundowning mediante la actividad física en las personas de edad utilizándose el método Kabath de Facilitación Neuropropioceptiva.

\section{Conclusión}

El síndrome de sundowning se debe a la alteración del ciclo circadiano del sueño y zeitgeber identificado por la disminución de producción de melatonina a nivel de la glándula pineal que afecta el eje hipotálamo hipofisario suprarrenal.

Los grandes síndromes geriátricos que se relacionan al síndrome de sundowning son el deterioro cognitivo y demencia, la dependencia y los estados de fragilidad.

El fundamento neurociencias en síndrome de sundowning se realiza mediante la valoración geriátrica integral mediante la medición de parámetros bioquímicos (niveles de vitamina $D$, melatonina) estudios de radio imagen (resonancia magnética cerebral, SPECT) evaluación del estado cognitivo (minimental test de folstein modificado y GDS), evaluación fisioterapéutica por medio de la escala de Rockwood para detección de estados de fragilidad y scores para valoración de estados de dependencia (Barthel y Lawton).

Las personas con síndrome de sundowning y deterioro neurocognitivo deben tener un abordaje integral para brindar calidad de vida y soporte en todas las fases de la demencia apoyado de una evaluación exhaustiva y criterios desde las ramas neurocientíficas.

\section{Referencias}

Alvarado , A., Alvarado , P., Rojas , L., y Oviedo , P. (2019). Necesidades de cuidado en los pacientes con demencia y/o alzheimer: una revisión integrativa. Cuidarte , 1-18. https://doi.org/10.15649/cuidarte. v10i3.813

Assad, Ghani, Sulehria, Mansoor, y Ameer. (2017). Intensive Care Unit Psychosis-sundowning: A Challenging Phenomenon. Indian Journal of Critical Care Medicine, 52-53. DOI: $10.4103 / \mathrm{jjccm}$. IJCCM_390_16

García-Díaz, G. F. de M. (2020). La Neurociencia en la educación. Revista Académica CUNZAC, 3(1), 37-43. https://doi. org/10.46780/cunzac.v3i1.16

Llanes, C., Pastor , T., Monforte , J., Román , A., López , P., y Franco, M. (2019 ). Síndrome del ocaso o "sundowning". NUEVO HOSP, 27-33. file:///C:/Users/DIEGO/Desktop/info\%20para\%20aticulo/ Nuevo\%20Hosp.2019_XV(1)\%2027-33. pdf

Ravi, y Bass. (2021). Mecanismos circadianos en medicina. El diario Nueva Inglaterra de medicina, 550-561. https://www.binasss.sa.cr/febrero/34.pdf

\section{Sobre los autores}

Equipo de investigadores del proyecto "Abordaje multidisciplinario del síndrome de sundowning en deterioro neurocognitivo" de la Universidad de San Carlos de Guatemala. 
Copyright (c) (2021) Héctor Alfredo René Bran Girón, Mario Josué Lopéz Rojas, Josué Elizandro Pinto Cabrera, Nataly Mercedes Chó Paiz, Ingrid Estefanía Linares Pinto y Edwin Orlando Lucas Vanegas

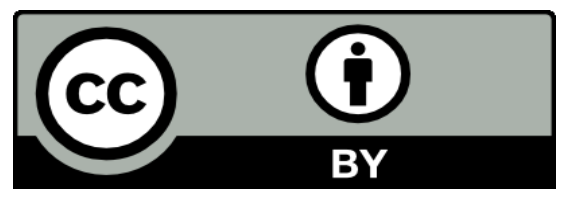

Este texto está protegido por una licencia Creative Commons 4.0.

Es libre para compartir, copiar y redistribuir el material en cualquier medio o formato y adaptar el documento, remezclar, transformar y crear a partir del material para cualquier propósito, incluso comercialmente, siempre que cumpla la condición de atribución: debe reconocer el crédito de una obra de manera adecuada, proporcionar un enlace a la licencia, e indicar si se han realizado cambios. Puede hacerlo en cualquier forma razonable, pero no de forma tal que sugiera que tiene el apoyo del licenciante o lo recibe por el uso que hace. 